Shachaf, P., \& Hara, N. (2007). Behavioural complexity theory of media selection: A proposed theory for global virtual teams. Journal of Information Science, 33(1), 63-74.

\title{
Behavioural complexity theory of media selection: A proposed theory for global virtual teams
}

Pnina Shachaf

\author{
School of Library and Information Science, $1320 \mathrm{E} 10^{\text {th }} \mathrm{St}$., LI005A, Indiana University, \\ Bloomington, IN, 47405-3907
}

\begin{abstract}
Noriko Hara
School of Library and Information Science, 1320 E $10^{\text {th }}$ St., LI025, Indiana University, Bloomington, IN, 47405-3907

th

Correspondence to: Pnina Shachaf, SLIS, 1320 E $10^{\text {th }}$ St., LI005A, Indiana University, Bloomington, IN, 47405-3907. E-mail: shachaf@indiana.edu
\end{abstract}

\begin{abstract}
This study proposes a behavioural complexity theory for media selection in global virtual teams.

This theory captures multiple contingencies into one holistic approach to media selection. Unlike existing linear and mechanistic theories of media selection, this heuristic theory moves away from the universal models that were previously proposed. The behavioural complexity theory assumes ambiguity and complexity of the media selection process in a nonlinear, organic, and holistic way. Behavioural complexity theory of media selection emphasizes the role of media repertoire, the ability of individuals to differentiate situations according to multiple contingencies, and their flexibility to effectively use multiple media in any particular situation. This theory is examined in a context of exploratory case study of global virtual teams' media selection in one of the leading fortune 500 corporations.
\end{abstract}

\section{Introduction}

Media selection theories focus on the choice of communication channels - given the choice, which media would one choose to accomplish a certain task? For example, one might prefer to use email to convey unpleasant information [1]. Past studies found that the perception of media selection and task technology fit differ in various cultural contexts $[2,3,4,5]$, yet traditional theories of media selection treat culture either as a contextual variable or as another social variable. This effect of culture is particularly instrumental in the context of global virtual teams (GVT), which are heterogeneous and internationally dispersed teams that rely mainly on information and communication technology to conduct their shared tasks [6]. Our study of interviews with 41 members of GVTs working in a leading Fortune 500 corporation emphasized this effect of culture on media choice. Their media selection for intercultural communication is complex and at times even paradoxical. These kinds of choices do not entirely correspond with the traditional approaches of media selection theories, which explain the choices by either rational or social models. The following quotes challenge the existing rational decision-making models and could not completely be elucidated by social models of media choice, especially in the context of GVTs. While the quotes do not contradict traditional media choice theories each of the theories only provide partial explanation for the complexity of media choices; none of these rational and social models provide a holistic explanation of media choice. In order to bridge this gap this study proposes a behavioural complexity 
theory for media selection in global virtual teams. The behavioural complexity theory provides a holistic explanation to media choices.

(1) So, I think if you go from a western kind of culture to some of these other ones that are different ... your communication style, and the medium you use would change...(2, $7 / 24 / 02$, U.S.)

(2) I use different [media] depending upon the situation. For example, when I'm working with Japan... I resort to e-mail quite often... (4, 7/29/02, U.S.).

(3) With people who don't speak very good English ... if I can, I prefer to use the written word, because you can be more concise, and it gives the person the ability to read it and understand it. $(23,9 / 23 / 02$, U.S.)

These quotes focus on challenges of cultural diversity that have been mediated by the use of media channels which were intentionally selected for this purpose. Quotes 1, 2, and 3 suggest that the leanest communication channel, e-mail, was selected for extremely complex communication incidents (intercultural communication) unlike the perceived best fit-face-to-face for complex and ambiguous tasks - according to media richness theory. Further, because these team members did not share the same social background and had to cope with multiple discontinuities [7], traditional social models $[23,24]$ were not useful for this particular situation because their assumptions were not met. These interviewees do not describe their choices as a universal way for GVT contexts. At times they had no idea what the social norms are in their team-mates' (remote) site and (different) cultures. They made assumptions about the situation and learned from their own best and worst experiences. Furthermore, unlike most media choice theories that approach media channels as distinct channel units (e.g., face-to-face, phone, e-mail), Quote 4 describes how multiple communication channels (e.g., e-meeting and teleconference) are used at the same time; channels complement each other in order to overcome intercultural communication challenges. Using only one of the available media channels increases intercultural miscommunication, but combining channels balances these limitations and improves intercultural mediated communication.

(4) We ... do ... an e-Meetings. . . share part of my desktop with someone else... this is typically what we set up when we talk with the [Japanese] guys... we'll show the agenda [on the screen]. As we talk [teleconference] about issues, we'll type in the result or whatever, the resolution, and we save all this stuff in a database, which we all have access to. . . . As we come to a resolution, I'll start typing there, and I'm hoping that that will help them verify the result, and that we're communicating effectively so they can see me typing the result on their screen. . . . Anything visual to sort of help guide the conversation or provide an outline that they can read, it helps them, it helps us all. (12, 8/30/02, U.S.)

Other descriptions made by GVT members have been predicted in part by traditional theories, such as media richness theory [8,9,10], task technology fit [11], social influence theory [12], and social presence theory $[13,14]$. These traditional media selection theories approach this process in an artificial and linear way that is characterized by a simple and predictable cause and effect relationships. Yet not a single theory had the power to provide a holistic explanation of the whole range of descriptions provided by our GVT interviewees. Unlike traditional approaches, GVT interviewees described a heuristic and nonlinear approach to media selection processes that assumes ambiguity and complexity. The Behavioural Complexity Theory (BCT) of media selection emphasizes the role of media channel repertoire, the ability of individuals to differentiate situations 
according to multiple contingencies, and their flexibility to use multiple channels in any particular situation. Channel repertoire is the range of media channels that can be used by GVT members to communicate with each other. We suggest here that BCT provides a more accurate description of media choice process in the context of GVT.

Following the description of our method in section 2, we will begin by outlining the theory of media selection (BCT) that we propose, along with its assumptions and main components, in section 3. Next, in section 4, we will revisit the traditional media selection theories and demonstrate how these theories, when they are applied in the context of GVTs, do not meet their assumptions. Data will be used to support our arguments, dismantle existing theories, and emphasize the main components of the BCT.

\section{Method}

Assuming that media selection by GVT members is fundamental to the completion of their tasks-because these teams rely mostly (and in some teams solely) on information and communication technology to conduct their shared tasks - this paper focuses attention on the media choice process as perceived and described by these team members. Data were collected as part of a larger exploratory study that aimed at understanding the impact of cultural diversity and information and communication technology on GVT effectiveness [6]. Using a case study approach, this paper aims to explain the media selection process of global virtual team members. The purpose of a case study is to "make observations about the explanatory power of different theoretical arguments that, through replication, can be argued to generalize." [15, p. 126]

The source of data was individual interviews with global virtual team members in a Fortune 500 corporation in the computer industry. The bulk of the data came from members of global virtual teams who worked in that leading multinational corporation. The multinational corporation's top management was based in the U.S. but a number of divisions were located around the globe. The corporation has employees in Asia, Europe, North and South America, and Australia. Theoretical sampling of participants for this study used the snowball sampling method. The sample size was finalized during data collection [16], with data collection ending as theoretical saturation was attained. In total, this study included 41 participants, forming a group that included individuals from nine countries of residency (France, Germany, Israel, Italy, Japan, Netherlands, Switzerland, U.K., U.S.), with numbers per country ranging from one to fifteen participants. The data resulted from nine months of data collection from June 2002 until February 2003 and originated from 41 interviews16 face-to-face and 25 via telephone. Due to the exploratory approach of this study, the interview protocol was developed with open-ended questions [17]. While face-to-face interviews were conducted to gain rich data, telephone interviews were used when face-to-face interviews were not possible and when access to interviewees was difficult or impossible due to geographical or time constraints [18]. This approach enabled the study to reach a sample population that was distributed in geographically dispersed locations.

Interviewees communicated with each other using multiple technologies in addition to faceto-face meetings. The media channels were mainly employed through corporate-wide use of Lotus groupware. Lotus groupware provides support for e-mail, Sametime (chat and e-Meeting), team room (shared electronic workspace), and other applications. Participants of this study reported on their use of e-mail, chat, e-Meeting (a web-based meeting using whiteboard, group chat, audio, video, and screen sharing), teleconference, and team room.

Following Miles and Huberman [17], the continuous process of data analysis was intertwined with additional data collection. Conceptualization and theory generation occur through a process of continuous data collection and data analysis [16]. The coding and interpretation made on early transcriptions were used during later interviews for data collection. An inductive approach was used 
for interpretation to enable the formation of grounded theory [16, 17]. Categories were developed from the data. Then concepts from the data were sorted 
according to the categories; an examination of the sorted data identified patterns and relationships. Data analysis and coding began once the first interview was transcribed and lasted until after the final interview was transcribed.

Data analysis was supported NVIVO 1.3, which is designed for qualitative analysis. This software was used not merely as a tool to mechanize the clerical tasks of data administration and archiving, but rather as a tool for analysis [19]. The code-and-retrieve approach retrieves all instances of data quickly with its cross-categories search option [20]; the co-occurrence of codes is valuable in the building of grounded theory. We used NVIVO 1.3 and not other software for qualitative analysis because it supports data transcribed in languages other than English (a portion of the data in this study is transcribed in Hebrew fonts).

\section{Behavioural complexity theory}

We propose a new theory of media selection: the behavioural complexity theory (BCT). We argue that BCT better explains the process of global virtual team members' media selection. This theory is composed of two elements: (1) repertoire of channels and (2) flexibility of individuals. According to the BCT, media selection is a process of excluding channels from the repertoire of media channels. Multiple contingencies affect this process of excluding channels; the process is reiterative until chosen channels are utilized. Instrumental to this process is individuals' flexibility to make complex and paradoxical selections (of one media channel, a range of channels, or at times, a combination of channels simultaneously) and to use channels in complex and paradoxical ways. We will first outline the BCT assumptions, then we will describe the role of channel repertoire along with the multiple contingencies that are involved in the process, and we will end with describing the flexibility of individuals to use channels in complex as well as paradoxical ways.

BCT assumes that media choice:

1. is context dependent; context involves the socio-cultural and technological environments.

2. is a decision making process that can be done at the individual, dyad, or group level.

3. is not merely a linear and rational process, but an iterative process.

\subsection{Media channel repertoire}

Media selection occurs among a repertoire of channels, which includes the range of channels adopted and used by team members for communication and information sharing. For example, most organizations today offer the option to communicate face-to-face, via phone, snail mail, memos, email, and some organizations offer support for additional communication channels, such as chat, team rooms, videoconferencing, and the like. Six contingencies limit this range of channels and are listed below:

1. Team geographical dispersion and multiple time zones.

2. Social proximity and cultural diversity.

3. Task at hand.

4. Individual preferences to use or avoid media channels. 
5. Accessibility of team members to use a particular media channel in a specific situation.

6. The initial channel that was used by a team member.

Despite the fact that these contingencies have, in part, been identified before $[3,17]$ we will discuss each of them. In doing so, we will support them with quotes from the data and will show that each contingency contributes to the "exclusion" process of limiting channel repertoire.

\subsubsection{Contingency 1 - Team geographical dispersion and multiple time zones.}

First, communicating across different time zones limits the range of channels. When communicating across different time zones, asynchronous channels, such as e-mail, are used (see Quote 5). When members are geographically dispersed, a limited range of channels are used in the process of selection, excluding, for example, face-to-face meetings. When time zone differences are wider, the range diminishes and synchronous channels are excluded during portions of the working day. For example, when a team member in the US (East Standard Time) wishes to communicate with a Japanese teammate, synchronous channels are almost always excluded from the repertoire of channels due to the thirteen hours difference between the two time zones. Since European and American team members can communicate only during their working hours (America's morning and Europe's afternoon), the repertoire of channels is limited to asynchronous channels or to synchronous channels only during several hours of each working day.

(5) Recognize that there's a [time zone] problem there and do what you can to overcome it. So with Japan I use a lot more e-mail because it works well over the time zone differences. A lot more e-mail there. (4, $7 / 29 / 02$, U.S.)

\subsubsection{Contingency 2 - Social proximity and cultural diversity.}

Quotes 6, 7, and 8, below illustrate how the initiator's perceived social proximity among people in the communication incident limits the range of channels. Social proximity in this context is defined by organizational vertical and horizontal differences, country of origin and country of residency differences, shared history, level of familiarity, and shared native language. Social proximity influences the preferred formality and synchronicity of the channel. For example, when members share high social proximity (close to each other) a wider range of channels can be used. Synchronous and informal channels (e.g., chat) are excluded from the repertoire and the range of channels is smaller when low social proximity among members is involved. Similarly, when vertical or horizontal organizational differences are significant, employees who are lower ranked or dispersed are more likely to initiate (upward) communication via formal and asynchronous channels. In a similar way, communication among pairs who do not share history and are unfamiliar with each other lead to selecting channels that are perceived to be widely adopted and used, such as e-mail and telephone. Consequently, social proximity limits the range of channels for GVT members. Quotes 1, 2, 3, and 4 above also illustrate how language and culture limit channel in addition to those below.

(6) The level of familiarity we have will have some effect. Most of the time if it is someone we never talked to, or it is the first time, or we are distant from each other, in a different hierarchical level, in other words he is a top executive or alike, or due to any other reason that creates distance, then I would rather use e-mail since it is more formal; the language is more formal. I can use the spell checker...If it is someone closer to me, closer might be that we 
have been talking a lot, or we are at the same league, or that we share physical proximity, or due to any other reason that makes me feel that this person is closer to me, I could easily use the chat. $(16,9 / 10 / 02$, Israel)

(7) ... if I'm communicating with someone in corporate that I've never met, it's unlikely that I'm just going to Sametime them. If it's someone up the chain, I'm going to do that appropriately in e-mail or call them on the phone. I'm not just going to Sametime them and say, "By the way, I'm so and so." (31, 10/21/02, U.S.)

(8) I wouldn't Sametime quite so much in a formal context, so let's say one of the high-level managers over in Japan came and said, "We need to know something about next year's planning cycle. How many people do you think we need to allocate?" I probably wouldn't use Sametime with that because it's too informal. There, they're really requesting some kind of formal statement. Then I would use e-mail...If there's some formal communication...Y You'd want a document so you can sit down and review it; you can look at it, you can think about it. You can't review that with a piece of speech. (4, 7/29/02, U.S.)

\subsubsection{Contingency 3 - Task at hand.}

The task at hand is another contingency that affects the channel that is selected. Some tasks are too complex to be communicated via particular channels. Thus, the range of channels for complex tasks is more limited. A wider range of channels can be used for routine tasks. Quote 9 extracts how the task at hand affects the selection.

(9) ...for instance if I have a quick question I would send them a chat. If that question would then have a complicated answer, I would pick up the phone. Because when you need to communicate and be clear and be sure that there is clarity, you talk [by] the phone. (2, 7/24/02, U.S.)

\subsubsection{Contingency 4 - Individual preferences to use or avoid media channels.}

In addition, the preferences of each individual influence the media choice. For example, a participant referred to a case "with people who don't read their e-mail, you don't send them an email." (31, 10/21/03, U.S.) The same interviewee (Quote 10) suggested that he will not use particular channels with specific individuals. Similarly, the channel selected can be limited based on specific individual preferences in a particular situation (Quote 11). It can be a joint process by the two people involved in the communication incident.

(10) Depending upon the individual, you'll come to a choice. I don't tend to treat groups as being all identical; I treat them as individuals, really, so different people different things suit. I can name names in the building where I know they actually prefer to use Sametime over face-to-face..." $(4,7 / 29 / 02$, U.S.)

(11) ...it's more to do with individual circumstances. There are other situations, obscure situations, where you might not do that... We have a lot of people working out of offices with no walls. In those circumstances, talking on the phone can be worse because you don't want people to overhear you. So in those circumstances, you might take it the other way. Even though the communication is not as easy, it's more private to do it through e-mail or 
through Sametime...I can then respect that, and say, "Well, do you want to find a private room?" or "Do you want to take this to Sametime?" (4, 7/29/02, U.S.)

Further, cultural preference and technology penetration rates affect the channel that one selects to communicate with someone in another country and further limit the range of channels that can be used. Quotes 12 and 13, made by two American interviewees, provide examples of perceived differences in the use of technology by European and Chinese users.

(12) Most of western Europe...much more pervasive with cell phone technology than we are. . . saying, "Here's my cell phone number, call me anytime." And they're very conducive to taking that call and transacting business, etc. But most American audiences are not as-even if they may have cell phones - are not as comfortable. .. oddly enough, if you ask them to check e-mail at a certain time, they're not going to do that, whereas we're quite comfortable doing that, but they're quite comfortable taking a cell call. . . I also think it may be somewhat cultural. $(7,7 / 30 / 02$, U.S.)

(13) You may not be able to get them [Chinese] on instant messaging, because they may not have as much access, so it's easier to get to them on the phone. They do have a lot of cell phones, so it's easy to get them on the phone. $(23,9 / 23 / 02$, U.S.)

3.1.5 Contingency 5 - Accessibility of team members to use a particular media channel in a specific situation.

Quotes 14 and 15 illustrate that accessibility of someone to respond (or not) in a particular moment limits the range of channels and determines channel choice. These interviewees reported that they tried several channels after the first attempts to use a channel were not successful. This process suggests that rather than a universal fit of one channel for one task, "best fit" of media is done by combining multiple channels (a repertoire of channels was selected) for one task. Moreover, media selection process involves several iterations of selecting appropriate channels; each attempt begins when the receiver is not accessible to use a channel. This process involves several channels in the repertoire until the message is transferred. A default channel (e-mail or voice mail) is used on occasions when the cost of effort (in terms of time) to use a channel accessible for both is too high for the particular task.

(14) ... we Sametime a lot, and we'll also use the telephone... if I call, and there's no response on the phone, I'll check the network to see if they're on Sametime. If they're there, I'll try that. If I don't get anything there, then I'll look to see if they have a cell phone. (11, 8/30/02, U.S.)

(15) ...if someone is on the phone and you call them you couldn't reach them, and you see that they are there on the Sametime, you use the Sametime ... interrupt them since they may be only listening and not presenting [at the teleconference]. And you send them quick Sametime or quick chat. (2, 7/24/02, U.S.)

3.1.6 Contingency 6 - The initial channel that was used by a team member.

Quote 16 suggests that the use of the initial channel is yet another contingency that affects the choice of channel to respond. Thus, the process of media selection in initial communication is slightly different than that of providing feedback and replying to a message. Often times team 
members are using the initial channel to respond to the sender, even though it became clear that this is very ineffective approach to accomplish a task. Since many of the communication incidents involve several exchanged messages, most of the messages are transferred back and forth by the initial channel. That is, the initiator of the communication incident selects a channel and the following messages are conducted with the same channel, even when it is not the best (or even good) fit with the task. Alternatives are not evaluated until it is evident that the used channel is ineffective, and then best fit is identified. This ineffective channel is, then, excluded from the repertoire of channels to be used.

(16) I even had [employees] who would... send e-mail, just e-mail after e-mail after e-mail on something that was very complex, arguing with each other, and they would actually sit next to each other in the office, and they wouldn't go talk to each other... (31, 10/21/02, U.S.)

Most of these contingencies have been identified in the literature [e.g., 3, 15, 21], yet previous theories assume that these contingencies help select one best channel for a particular situation. We found that these contingencies are instead limiting factors; they affect the exclusion of channels from being used in a particular situation. In other words, in other theories, like the media richness theory, the approach to the contingencies have been positive (or best fit) whereas in our proposed new theory, BCT, it is a reflection of an opposite process (unfit), reducing, excluding, and eliminating channels from the repertoire.

\subsection{Individual's flexibility}

The second component of the new theory that we propose, BCT, involves individuals' flexibility to use multiple channels in a particular situation. Hooijberg et al. [22], Boal and Hooijberg [23], and Kayworth and Leidner [24] discussed the application of behavioural complexity theory to explain leadership behaviours. Similarly, we propose that the media selection process involves an adjustment process according to the limitations that are made by the specific contingencies. Effective channels selection involves the individuals' ability to differentiate situations by multiple contingencies and to select and use channels for a particular situation. The complex and dynamic social context of global virtual team members forces individuals to be flexible in their media selection. Their flexibility enables them to adapt their behaviours according to multiple contingencies; these affect their complex choices of communication channel and at times results in paradoxical selections in response to the environment. An individual's flexibility is instrumental in adapting to the dynamic environment. These adjustments result occasionally in paradoxical media choices. One example of the paradox is illustrated by the case that team members choose lean media for a complex task-heterogonous teams choose channels that are inconsistent with task technology fit. Although GVT members are aware of task technology fit - for certain tasks it is more effective to use a particular communication channel - they were able not only to fit a channel that is not the best fit, but also to report on their paradoxical choices as "best fit."(Quotes 1-3) Specifically, the paradoxical best fit involves reports on e-mail, a lean channel, as the preferred "best fit" to almost any intercultural communication incident, which is a complex task [4]. Further, face-to-face meetings are the richest channel, according to Daft and Lengel $[8,9]$ and to Sproull and Keisler [14]; yet Shachaf [4] reported on more (intercultural) miscommunication that occurred at these meetings than when communicating via e-mail. The interviewee in Quote 17 illustrates and describes difficulties understanding team-mates during face-to-face meetings compared with understanding them via email. 
(17) Once a group learned how to work via e-mail, they experience difficulties every time they meet face-to-face...via e-mail there is focus; they know to express exactly what they wish to say. With face-to-face, when one is free from this focus, then...the entire communication is out of balance, people say chunks of sentences...you are expecting people that you are communicating with to behave in a certain way, and the observed behaviour does not fit the written style...people that express themselves very precisely in writing are very unorganized; they could be very unorganized when they talk. You never know how long it takes them...how much time they put into...or that they have learned how to express themselves in writing and talking is difficult for them. Anyway, in any face-to-face meeting, you spend about a half of a day experiencing communication difficulty, because people don't understand each other. (32, 10/22/02, Israel)

In addition to this paradox of task technology fit, GVT members also used technology in a variety of complex ways. For example, Quote 4 shows that multiple channels are simultaneously used in a complicated situation. Complex situations, such as routine teleconference meetings among culturally diverse and geographically dispersed team members, predefined the combination of channels. For example, the use of teleconference and e-Meeting during team meetings enabled team members to overcome predictable and intricate intercultural miscommunication incidents. In particular, GVT members were aware of the difficulties in understanding each other over the phone, due to the reduced cues and increased miscommunication due to accents and pronunciations. It was recognized by these team members that the written language of non-native English speakers is better than their spoken language and that their ability to understand written text is also better than their ability to process the spoken language. For that reason, they combined these channels into a sophisticated use of available communication channels.

Thus, it is clear that individual team members are flexible in their media use for multiple tasks in a complex and paradoxical ways. This flexibility improves their ability to perform their shared tasks.

To sum up, the new theory that we propose here, BCT, provide a holistic explanation of media choices of GVT members. It is composed of a process of excluding channels from media channel repertoire, according to six contingencies. Multiple channels are considered to be used individually or in combination with other channels. Individuals' flexibility to use multiple channels and their use of these channels effectively in complex and at times paradoxical ways is another component of the BCT. The next section focuses attention on revisiting traditional media selection theory and demonstrates the weakness of the explanations of this process by the existing theories.

\section{Media selection theories revisited}

Media choice of managers and employees was the focus of studies and theories for decades. The major theories of media selection to be revisited are media richness theory $[8,9,10]$, task technology fit [11], social presence theory [13,14], and social influence theory [12]. These classical theories are cited by hundreds of empirical and theoretical papers, many of which involve ICTs.

Media richness theory is a contingency theory, which suggests that the more information a medium conveys, the richer the communication is $[8,9,10]$. The capacity of the information channel is influenced by four criteria: the ability to simultaneously handle multiple cues, including physical presence, voice inflection, body gesture, words, numbers, and graphic symbols; the ability to establish two-way communication and receive instant feedback; the ability to establish personal focus; and the ability to use language variety. Findings suggest that managers prefer rich media for ambiguous communications and leaner media for unequivocal messages [8]. While this theory was developed originally without references to ICTs, ICTs were integrated later. Researchers have long studied the effect of media richness on media choice and the effects of media use on team 
performance [25, 26]. Hollingshead, McGrath, and O'Connor [11] modified the contingency framework and further elaborated on task technology fit. Their task technology fit matrix has two axes: the type of task and the type of media channel. There are varying patterns between the information richness of the media and the information richness needs of the tasks. Groups may be less efficient if the media is too rich or too lean for the task at hand.

Yet, interviewees described their choices in a manner that challenges the assumptions on which these theories rely. Media richness theory and task technology fit models assume that selection corresponds to one technology, at one time, for one task. These theories assume that innate media characteristics are matched to the richness of a task and that this fit is universal. They also assume that selection is a rational decision-making process, where individuals identify the need and potential channels they can use, evaluate each one, and then select one. However, the interviews suggest that (a) the choice of a channel is not purely rational but rather satisfying (use of first channel available); (b) the richness of the channels is not the only consideration that defines its fit to the task - multiple contingencies are also involved in this process (such as the social context); and (c) the fit is not merely of one channel for a task but of multiple channels (repertoire of channels) to a task. These three assumptions that have not been met in the context of media choices of GVT members are supported with interviewees' quotes.

(a) Quotes 14 and 15 suggest that the choice of a channel is not purely rational but rather satificing (use of first channel available) [27, 28]. These interviewees explain hypothetical media choices they make when trying to communicate with someone else. The first choice was the phone, but this was not available, the next attempt was chat (using Lotus Sametime), which is a staisficing channel, and the third choice was the cell phone. Quote 14 illustrates a process by which the channel is clearly not the rational choice (it is not based on rational evaluation of all existing options), but the use of the initial channel that triggered the discussion, and as long as this was still satisficing, the conversation went on using this channel (see also Quote 16).

(b) Further, interviewees' descriptions support that the richness of the channels is not the only consideration that defines its fit to the task, but multiple contingencies are also involved in this process. Six contingencies were described above, and these include, in addition to the task at hand, the initial channels, physical proximity and time zone, social proximity and culture, accessibility, and individual preferences.

(c) The final assumption that media richness theory makes and which is not met here is that the fit is not merely of one channel for a task but of multiple channels (repertoire of channels) to a task. Quotes 14 and 15 suggest that a few channels are concurrently considered as "fit" for the task at hand. The interviewees describe how they try to communicate a message in one, two, and three channels (each at a time), and considered each to be appropriate for their communication task. Furthermore, the interviewee in Quote 4 explained how he uses a combination of channels at the same time. Additionally, Quotes 5 and 6 implied "multitasking" in the concurrent use of multiple media channels with using the phone and chat as side conversations.

When GVT members use information and communication technologies or meet face-to-face, they use a combination of channels at the same time. The use of channel combinations is evident at both the individual and group levels. For example, individual team members use chat while talking on the phone or during face-to-face meetings; groups use e-Meeting during their teleconference meetings. The former enable individuals to be multitasking and the latter, by using more than one channel for communication, as one complements the other, creates new capabilities for communication effectiveness. This type of channel combination use (Quote 4) corresponds to the 
idea of "beyond being there" presented by Hollan and Stronetta [29]. They suggest that one of the ways in which electronic media may be better than face-to-face, and not merely an imitation of faceto-face, is by combining channels. Channel combinations, such as e-Meeting and teleconference, is another example of this idea of "beyond being there."

To sum up, while media richness theory and task technology fit are well cited in previous studies, the assumptions on which they are based are not met in the context of media choice of GVT members.

Furthermore, Rice, D'Ambra, and More [3], based on media richness theory, conducted a cross-cultural comparison of organizational media choices and found that only with telephone and email did cultural perceptions of media richness correlate with the theory. Other media channels (faceto-face, business memo, and voice mail) had no cross-cultural agreement regarding degree of media richness. Preferences for face-to-face and telephone correlated with message equivocally. Participants from collectivist cultures rated the telephone as less rich and the business memo as richer than did participants from individualist cultures. Like previous studies, Rice et al.'s [3] research on media choices in a global context did not fully support media richness theory. Rice, et al. [3], while focusing on cross-cultural differences and similarities in intracultural communication, suggested in their conclusion that differing perceptions of media richness would complicate intercultural communication. "Many elaborations [to media richness theory] have been suggested . . . such as situational constraints, symbolic uses and values, initiator-receiver distinctions, sequences of media use, effects of time and experience, and social information-processing."[3, p. 21]. Further, Robert and Dennis [30] emphasized the paradox of richness effects on media use and claimed that "[t]he paradox of richness lies in its duality of impact: from a cognitive perspective, rich media high in social presence simultaneously acts to both improve and impair performance." [p. 10]

Another media choice theory is the social presence theory, which states that the higher the capabilities of the medium are to convey visual and non-verbal cues, the higher the social presence of individuals will be [13]. Social presence refers to the degree to which a medium allows a user to establish a personal connection with others. A high presence medium (face-to-face) is rated more personal, sociable, and warm, unlike a low presence medium (e-mail), which is less personal and less sociable. The theory suggests that the level of social presence needed for a particular communication incident determines the medium. The theoretical foundation of social presence draws from Daft and Lengel's $[8,9]$ media richness theory. Social presence, which is affected by the communication channel, is a subjective perception of the interaction realness in the communication incident. Based on the social presence theory electronic media channels are leaner than traditional face-to-face and phone channels. The lack of social cues, lack of context (physical environment), and lack of nonverbal behavior reduce both the static and dynamic cues of the communication. Interactions are more impersonalized and task oriented when e-mail is used. Further, the decrease in social cues has a deregulating effect on communication. Specifically, people overestimated their contribution as well as the number of messages they received. Other effects of reduced social cues are status equalization and uninhibited behavior-such as flaming, communicating bad news, and flouting social conventions [14].

Therefore, richness or leanness is not an inherent property of e-mail (as perceived by media richness theory), but an emergent property of the interaction of the e-mail medium with its organizational context [31]. Accordingly, managers are not passive recipients of data, but active producers of meanings. Likewise, Markus [15] emphasizes that the social context is more important than the medium itself for communication effectiveness. Even a lean medium can be rich if the organizational context supports it.

Another effort to cope with the anomalies in media richness theory was made by Schmitz and Fulk [12] who proposed a social influence model of technology use. The model suggests that media 
perception is in part socially constructed; media choice is affected not only by the characteristics of the channel itself but also by the perception of the media channel. The theory predicts that media choice will be affected by individual preferences and also to a certain extent by the social process.

The social influence model and the social presence theory suggest a subjective process of media selection. They suggest that the social context of individuals and groups influences their media selection. It assumes members' homogeneity within social groups and heterogeneity among different social groups. However, global virtual teams (GVT) are geographically dispersed, culturally diverse, and heterogeneous. Thus, this assumption was not confirmed. In reality, the two unique characteristics of GVT, geographical dispersion and cultural diversity, create discontinuities among team members [7]. The social context of GVT members is not shared across multiple sites and countries. Individual team members act according to the impact of multiple social environments and develop, to a certain extent, their own team social context. Thus, GVT members behave in multiple ways when it comes to their use of media channels that cross team boundaries.

Although there is not a strong empirical support to these social theories, the socio-cultural context of GVT should not be overlooked. Media perception varies among team members from different countries and media selection also varies when intercultural communication is involved [4]. A few studies on (dispersed) virtual teams have also focused on the process of media selection. First, reports supporting media richness theory suggested that virtual team members tended to use face-toface and telephone for the more ambiguous tasks and synchronous collaborative technology for more routine tasks. However, later, Maznevski and Chodoba [21] observed that team members were able to adjust to technology for more ambiguous tasks as well. For example, brainstorming was conducted in face-to-face meetings during the first stages of teamwork but later on was accomplished using collaborative technology. Although media choices were consistent with media richness theory, the fact that researchers did not find an exact correspondence between message characteristics and media choice suggests the importance of the role of social context in media selection and the effect of the structural characteristics of the team. Maznevski and Chodoba [21] claimed that the higher the task interdependence, the more communication incidents are expected to occur. They argue that because message complexity increases with the number of borders, cultures, organizations, and professions spanned, communication requires richer media when crossing such boundaries. These findings emphasize the need for a theory that can capture the complexity of media selection process in these multifaceted and ambiguous situations, of which global virtual team members are part. The new theory that we proposed here, BCT, aims to improve our understanding of this process among GVT members.

\section{Conclusion}

In an effort to provide a better understanding of media selection processes described by GVT members, we proposed a new behavioural complexity theory and discussed its assumptions and its components, i.e., channel repertoire and flexibility. We revisited traditional media selection theories and suggested that behavioural complexity theory has more explanatory power over traditional theories in that it captures the complexity and paradox involved in this process. We provide illustration of behavioural complexity theory that is based on a case study of GVT in a multinational corporation.

Specifically we claim that media choice is a process of elimination, excluding channels and limiting channel repertoire in a particular situation. This process is affected by six contingencies: physical proximity, task at hand, social proximity, sender and receiver accessibility to use a channel, individual preferences about a channel, and the initial channel.

Further we claim that the flexibility of individuals to use multiple channels for a particular task, and to use combinations of channels at the same time, is a critical adjustment process. The findings 
suggest a more naturalistic way of media selection in GVT contexts. Because communication is an essential part of working in GVTs, channels selection may affect team performance. The six contingencies suggested in the proposed BCT can be used to educate employees who work in GVT environments in order to perform their tasks more effectively. For example, although currently GVT members tend to continue using initial channel of communications, they may be more self-aware of the process and reconsider better channel or range of channels for communication. In addition, instead of multi-channel use evolving, GVT members could consciously choose to use multichannels for complex tasks. Furthermore, GVT members should be aware of the process in which channels are excluded from a repertoire of channels and should be flexible and able to effectively use a variety of channels in any particular situation. We hope that this research informs both practitioners and researchers who are involved in media selection for GVT contexts.

Future research should focus on the channel elimination process and on the utilization of multiple channels simultaneously. One of the limitations of this paper is that the theory was developed based on data that was collected from one organization. We hope that future studies will examine the transferability of this theory to other settings.

\section{References}

[1] N. Kumar and I. Benbasat, Para-social presence and communication capabilities of a website: A theoretical perspective, e-Service Journal 1(3)(2002) 5-24.

[2] A.P. Massey, C.Y. Hung, M. Montoya-Weiss, and V. Ramesh, When culture and style aren't about clothes: Perceptions of task-technology "fit" in GVTs. In: Proceedings of GROUP '01 (Boudler, CO, 2001) 207-213.

[3] R.E. Rice, J. D'Ambra and E. More, Cross-cultural comparison of organizational media evaluation and choice, Journal of Communication 48 (3) (1998) 3-26.

[4] P. Shachaf, Bridging cultural diversity through e-mail, Journal of Global Information Technology Management 8(2)(2005) 46-60.

[5] K. Steinwachs, Information and culture - the impact of national culture on information processes, Journal of Information Science 25(3)(1999) 193-204.

[6] P. Fichman-Shachaf, Global Virtual Teams: The Impact of Cultural Diversity and Information and Communication Technology on Team Effectiveness. (Unpublished doctoral dissertation, University of North Carolina, Chapel Hill, 2003).

[7] M.B. Watson-Manheim, K.M. Chudoba and K. Crowston, Discontinuities and continuities: A new way to understand virtual work, Information Technology and People 15(3)(2002) 191-209.

[8] R.L. Daft, and R.H. Lengel, Organizational information requirements, media richness and structural design. Management Science 32(5) (1986) 554-572.

[9] R.L. Daft, R.H. Lengel and L.K. Trevino, Message equivocality, media selection, and manager performance: Implications for information systems, MIS Quarterly 11(3)(1987) 355-366.

[10] R.H. Lengel and R.L. Daft, The selection of communication media as an executive skill, Academy of Management Executive 2(3)(1988) 225-232. 
[11] A.B. Hollingshead, J.E. McGrath and M. O'Connor, Group task performance and communication technology: a longitudinal study of computer-mediated versus face-to-face work groups, Small Group Research 24(3) (1993) 307-324.

[12] J. Schmitz and J. Fulk, Organizational colleagues, media richness, and electronic mail, Communication Research 18(4)(1991) 487-523.

[13] J. Short, E. Williams and B. Christie, The Social Psychology of Telecommunication (John Wiley \& Sons, New York, 1976).

[14] L. Sproull and S. Keisler, Reducing social context cues: Electronic mail in organizational communication, Management Science 32(11)(1986) 1492-1513.

[15] L. Markus, Electronic mail as the medium for managerial choice. Organization Science 5(1994) 502-527.

[16] B. Glaser and A. Strauss, The Discovery of Grounded Theory: Strategies for Qualitative Research (Aldine, Chicago, 1997).

[17] M.B. Miles and M.A. Huberman, Qualitative Analysis: An Expanded Sourcebook (Sage, Tausand Oak, CA, 1994).

[18] B.L. Berg, Qualitative Research Methods for the Social Sciences (Allyn \& Bacon, Needham Heights, MA, 2001).

[19] U. Kelle, Theory building in qualitative research and computer programs for the management of textual data, Sociological Research Online 2(2)(1997). Available at: http://www.socresonline.org.uk/socresonline/2/2/1.html (accessed 13 November 2003). 
[20] A. Coffey, B. Holbrook and P. Atkinson, Qualitative data analysis: Technologies and representations. Sociological Research Online 1(1) (1996). Available at: http://www.socresonline.org.uk/socresonline/1/1/4.html (accessed 13 November 2003)

[21] L.M. Maznevski and K.M. Chudoba, Bridging space over time: Global virtual team dynamics and effectiveness, Organization Science 11(5)(2000) 473-492.

[22] R. Hooijberg, J.G. Hunt and G.E. Dodge, Leadership complexity and development of the Leaderplex model, Journal of Management 23(3)(1997) 375-408.

[23] K.B. Boal and S. Hooijberg, Strategic leadership research: Moving on, Leadership Quarterly 11(4)(2001) 515-549.

[24] T.R. Kayworth and D.E. Leidner, Leadership effectiveness in global virtual teams, Journal of Management Information Systems 18(3)(2001) 7-40.

[25] A. Malhotra and A. Majchrzak, Virtual workplace technologies, MIT Sloan Management Review 46(2)(2005), 11-12.

[26] K. S. Suh, Impact of communication medium on task performance and satisfaction: An examination of media-richness theory, Information and Management 35(5)(1999), 295-312.

[27] H.A. Simon, A behavioral model of rational choice, The Quarterly Journal of Economics 69(1)(1955), 99-118.

[28] H.A. Simon, Rational decision making in business organizations, The American Economic Review 69(4)(1979), 493-513.

[29] J. Hollan and S. Stronetta, Beyond being there. In: Proceedings of the ACM Conference on Human Factors in Computing Systems (Monterey, CA, 1992) 119-125.

[30] L.P. Robert and A.R. Dennis, Paradox of richness: A cognitive model of media choice, IEEE Transactions on Professional Communication 48(1)(2005), 10-21.

[31] A.S. Lee, Electronic mail as a medium for rich communication: An empirical investigation using hermeneutic interpretation, MIS Quarterly 18(2)(1994) 143-157.

\section{Acknowledgment}

We would like to thank Alice Robbin for her insightful comments and would also like to thank the anonymous reviewers. 
1

The references for each direct quote consist of the identification number assigned to each participant, the date of the interview (month/date/year), and the participant's country of residence.

Since native English speakers were more articulate in English, most of the quotations used in this study are from interviews with native speakers.

2

The quotation was translated from Hebrew.

For translation reliability check the Hebrew text, which is provided here:

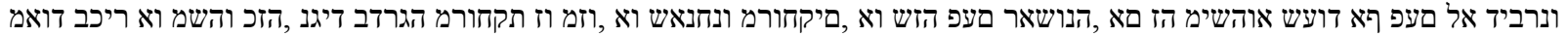

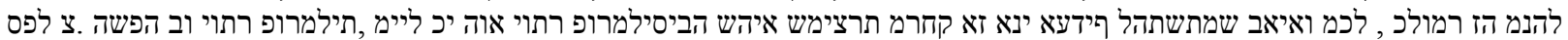

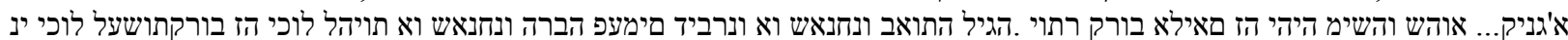

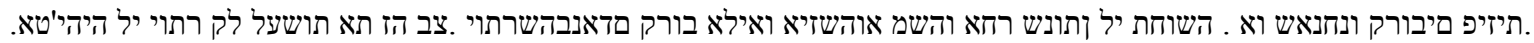

3

The quotation was translated from Hebrew. The Hebrew text is:

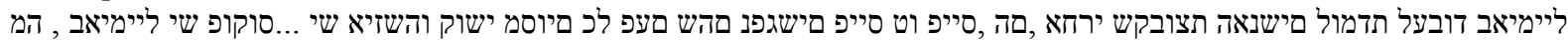

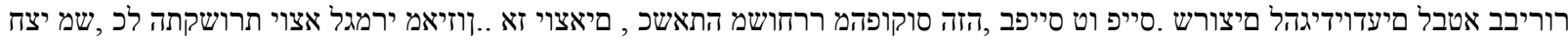

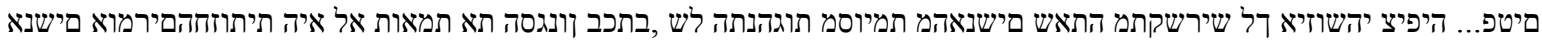

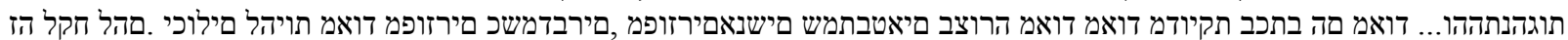

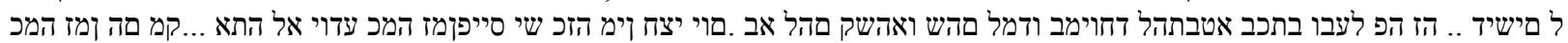

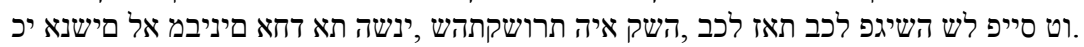

